\title{
Some properties of the curve of constant bearing
}

\author{
By D. Martin.
}

If $Z$ is a fixed point on the surface of the earth (assumed spherical) and $P$ is the North Pole, then the locus of a point $X$, which moves in such a way that the angle $a$ between the great circle arcs $P X, Z X$ is constant, is called a curve of constant bearing.

$a$ is measured clockwise from $X P$; it is then the great circle bearing of $Z$ from $X$ as defined in navigation, and lies within the range $0^{\circ}$ to $360^{\circ}$.

Curves of constant bearing are of some importance in navigation because, if a ship or aircraft at $X$ takes a bearing of a radio station at $Z$, the position line so obtained is an are of such a curve. Nevertheless, few properties of the curves seem to be recorded ${ }^{1}$; the reason is probably that practical navigators are interested not in the actual curves in their entirety but in the projections on a Mercator chart of comparatively short lengths of them.

In this note some simple properties of the curves are obtained; the derivation of the results is very straightforward and, needless to say, no originality is claimed.

We begin by writing down the equation of a curve of constant bearing. Let the latitudes of $X$ and $Z$ be $\phi, \phi_{0}$ respectively, and let the meridian of $Z$ be that of zero longitude; the longitude $\lambda$ of $X$ is considered as positive or negative according as it is Easterly or Westerly. Since, by convention, the angles of a spherical triangle cannot exceed $180^{\circ}$, two cases $a<180^{\circ}$ (fig (i)) and $a>180^{\circ}$ (fig. (ii)) must be considered. Then, in both cases, by the Four Part Formula of Spherical Trigonometry, the equation of the curve of constant bearing is ${ }^{2}$

$$
\cos \lambda \sin \phi=\cos \phi \tan \phi_{0}+\sin \lambda \cot a .
$$

A number of useful properties of the curve follow immediately from (1).

(a) The curves of constant bearing (of a given point) for bearings $a$ and $180^{\circ}+a$ form parts of the complete curve defined by (1). For

1 One or two are given in the Admiralty Manual of Navigation, Vol. III. (H.M.S.O., 1938).

2 Admiralty Manual, loc. cit., p. 203. There is a minor difference in sign due to our convention regarding the sign of the longitude. 
simplicity, we shall call this complete curve a curve of constant bearing.

(b) All curves of constant bearing pass through the North and South Poles; these are approached along the meridians of longitude $a$ and $180^{\circ}-a$ respectively. The bearing changes abruptly by $180^{\circ}$ as the curve passes through each pole.

(c) Every curve of constant bearing of $Z$ passes through both $Z$ and $Z^{\prime}$ the antipodal point of $Z$. The bearing changes discontinuously by $180^{\circ}$ as the curve passes through each of these points.

(d) All curves of constant bearing are centrally symmetric about the two points on the equator of longitude $\pm 90^{\circ}$.

(e) The curve for bearing $360^{\circ}-a$ is the image in the meridional plane of $Z$ of the corresponding curve for bearing $a$.

$(f)$ The points of intersection of a curve of constant bearing and the equator are given by

$$
\sin \lambda+\tan \phi_{0} \tan a=0,
$$

whence it follows that the longitudes of the points of intersection are supplementary. The points are real and distinct, real and coincident or imaginary according as $\left|\tan \phi_{0} \tan a\right|$ is less. than, equal to or greater than unity. In the last case, the curve consists of two ovals, one in each hemisphere.

$(g)^{1}$ If we change to Cartesian coordinates $x=r \cos \phi \cos \lambda$, $y=r \cos \phi \sin \lambda, z=r \sin \phi$, then (1) becomes

$$
z x=\left(x^{2}+y^{2}\right) \tan \phi_{0}+y r \cot \alpha .
$$

The curve of constant bearing is therefore the intersection of two quadrics (a sphere and a hyperboloid of one sheet) and is, in fact, a quartic curve of the first species.

We obtain next an expression for the angle between the curve of constant bearing at any point on it and the meridian at that point.

In fig. (iii) $X(\phi, \lambda)$ and $Y(\phi+\delta \phi, \lambda+\delta \lambda)$ are neighbouring points on a curve of constant bearing and $Y K$ is an arc of a great circle perpendicular to $P X$. Then

$$
\frac{Y K}{\bar{X} K}=\frac{\delta \lambda \cos \phi}{\delta \phi}
$$

and, if $\psi$ denotes the angle between the curve and the meridian at $X$ (measured clockwise from the meridian),

$$
\tan \psi=\cos \phi d \lambda / d \phi \text {. }
$$

1 Pointed out to me by Dr L. M. Brown. 
By (1);

$$
\frac{d \lambda}{d \phi}=\frac{\cos \lambda \cos \phi+\sin \phi \tan \phi_{0}}{\sin \lambda \sin \phi+\cos \lambda \cot \alpha},
$$

whence (1), (3) and (4) yield 1

$$
\tan (\alpha-\psi)=\sin \phi \tan \lambda \text {. }
$$

A consequence of (5) is that where the curve cuts the equator and the meridian of $Z$ (at $Z$ and $Z^{\prime}$ ), it is inclined at an angle $a$ to the meridian.

We next prove that the loci of the turning points in longitude and the turning points in latitude of the curves of constant bearing of $Z$ are great circles.

By (4), the turning points in longitude are given by

$$
\cos \lambda \cos \phi+\sin \phi \tan \phi_{0}=0 \text {. }
$$

Now the equation of the great circle with pole at $\left(\phi^{\prime}, \lambda^{\prime}\right)$ is

$$
\cos \phi^{\prime} \cos \lambda^{\prime} \cos \phi \cos \lambda+\cos \phi^{\prime} \sin \lambda^{\prime} \cos \phi \sin \lambda+\sin \phi^{\prime} \sin \phi=0 ;
$$

hence by (6), the turning points in longitude lie on the great circle with pole at $Z$.

Similarly, for the turning points in latitude,

$$
\sin \lambda \sin \phi+\cos \lambda \cot \alpha=0 \text {; }
$$

elimination of $a$ between this equation and (1) yields

$$
\cos \lambda \cos \phi \tan \phi_{0}-\sin \phi=0
$$

as the equation of the locus of the turning points in latitude. This represents a great circle with pole $90^{\circ} \mathrm{N}$. of $Z$.

The properties derived above enable us to sketch the family of curves of constant bearing (of $Z$ ) as in fig. (iv). The latitude of $Z$ has been taken as $40^{\circ} \mathrm{N}$. In following out any particular curve in the figure, it is useful to remember that the bearing changes abruptly by $180^{\circ}$ at $Z$ and $Z^{\prime}$, and at each pole.

When $\phi_{0}=0$ and $a=90^{\circ}$, the curve of constant bearing degenerates into two circles, the equator and the meridian of longitude $\pm 90^{\circ}$. Also if the earth were flat, a curve of constant bearing would, by reason of its definition, be a circle passing through $P$ and $Z$. It is natural then to enquire if the curve has any properties in common with the circle; so far I have been able to discover only two such properties.

1 Admiralty Manual, loc. cit., p. 204. 

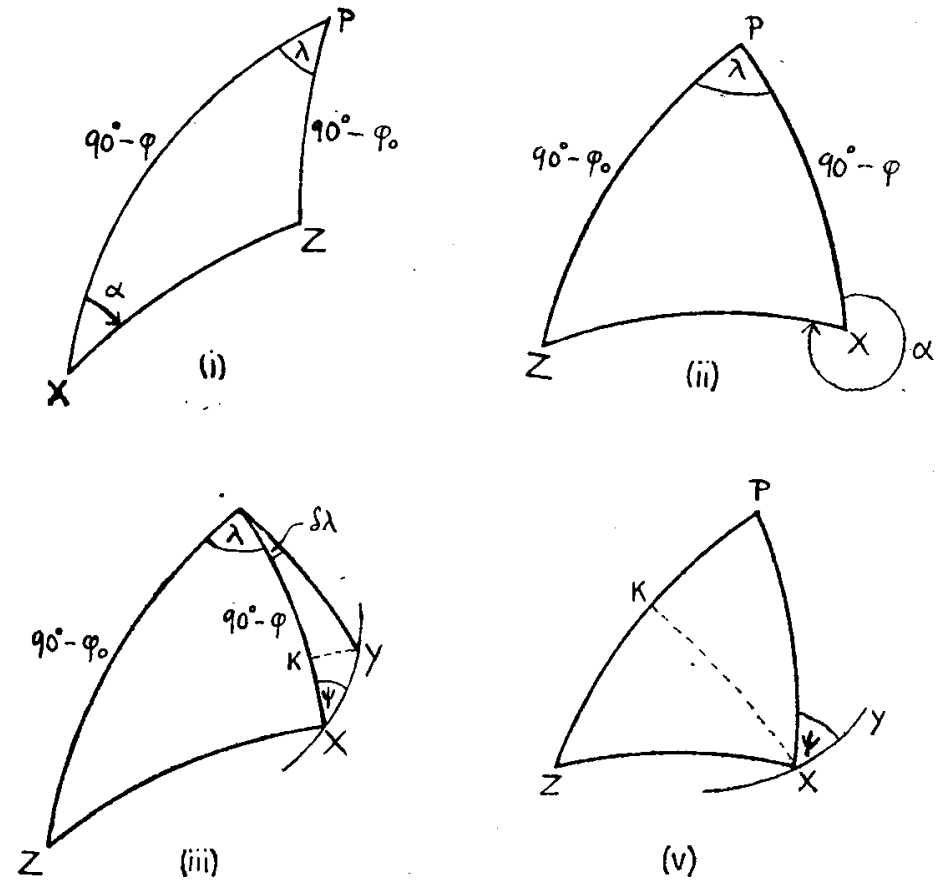

(v)

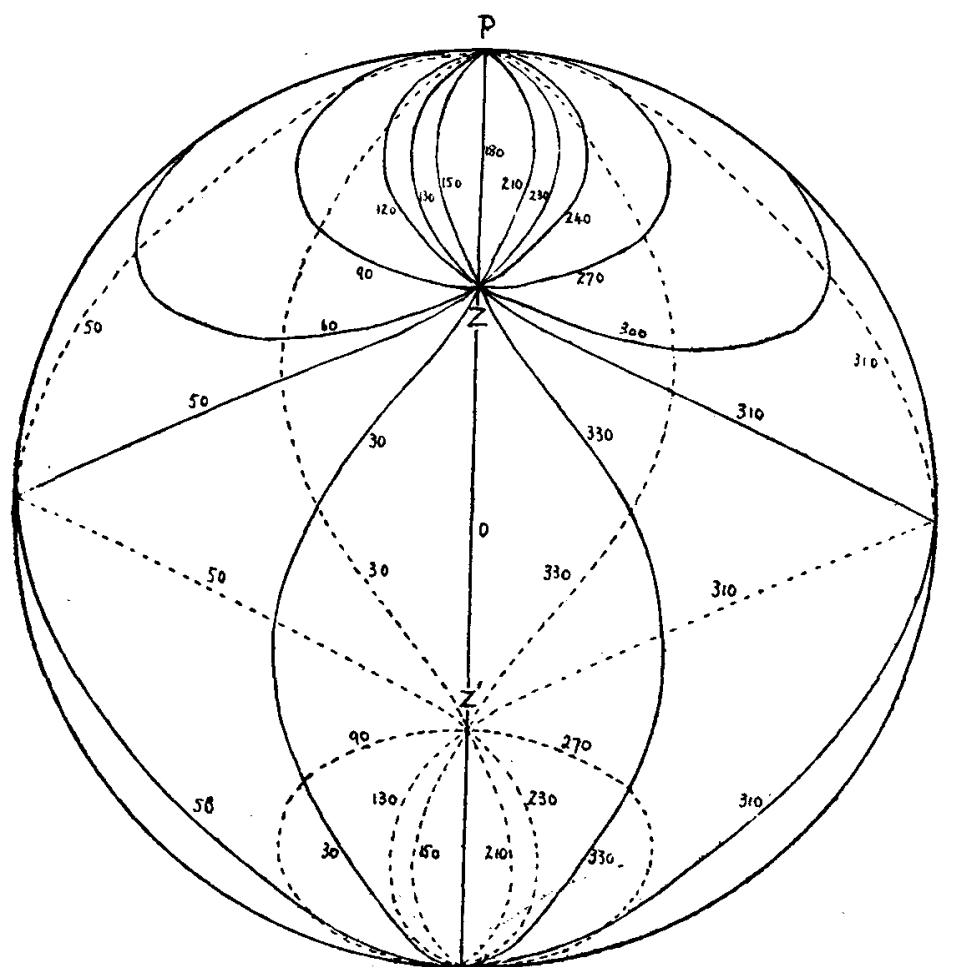


(A) In fig. ( $\nabla) X Y$ is an arc of a curve of constant bearing and $X K$ is an arc of a great circle perpendicular to $P Z$. Then, applying Napier's Mnemonic to the right angled triangle $P K X$,

$$
\cot P \hat{X} K=-\sin \phi \tan \lambda \text {, }
$$

$P \hat{X} K$ being measured clockwise from $X P$. Then, by (5)

$$
\cot P \hat{X} K=-\tan (\alpha-\psi) \text {, }
$$

whence, if we decide to take $Y$ on the opposite side of $X$ from $Z$ and measure $Y \hat{X} Z$ clockwise from $X Y$,

and

$$
\begin{aligned}
& \cot P \hat{X} \dot{K}=-\tan Y \hat{X} Z \\
& P \hat{X} K-Y \hat{X} Z= \pm 90^{\circ}
\end{aligned}
$$

as in the case of the circle.

(B) We consider next the orthogonal trajectories of the oneparameter family of curves of constant bearing, obtained by giving $a$ a fixed value.

By isolating $\tan \phi_{0}$ in (1) and differentiating, we find that $\cos \phi(\sin \lambda \sin \phi+\cos \lambda \cot \alpha) d \lambda=(\cos \lambda-\sin \phi \sin \lambda \cot \alpha) d \phi$.

Now

$$
\left(\frac{1}{\cos \phi} \frac{d \phi}{d \lambda}\right)_{o . T .}\left(\frac{1}{\cos \phi} \frac{d \phi}{d \lambda}\right)_{\text {C.C.B. }}=-1,
$$

whence we find that the differential equation of the orthogonal trajectories is

$(\cos \phi \cos \lambda-\sin \phi \cos \phi \sin \lambda \cot \alpha) d \lambda+(\sin \lambda \sin \phi+\cos \lambda \cot \alpha) d \phi=0 ;$ $\sec ^{2} \phi$ is an integrating factor, and the solution is

$$
\cos \lambda \sin \phi=C \tan \alpha \cos \phi-\sin \lambda \tan \alpha,
$$

where $C$ is an arbitrary constant. Comparing (9) with (1), we find that the orthogonal trajectories form the one-parameter family of curves of constant bearing, for which the bearing has the fixed value $90^{\circ}+a$. This result, which can easily be verified by means of (5), would also be true if the curves of constant bearing were circles.

From the analogy with coaxal circles, it might be conjectured that the orthogonal trajectories of the one-parameter family of curves of constant bearing, obtained by fixing $\phi_{0}$ instead of $\alpha$, would form a family of ourves of constant bearing passing through two fixed imaginary points and having imaginary values of $a$; but this does not seem to be so. The equation of the family of orthogonal trajectories turns out to be

$$
\sin ^{2} \lambda+\sec ^{2} \phi-2 \tan \phi_{0} \cos \lambda \tan \phi=C,
$$


A geometrical proof of taN $\frac{1}{2}(B-C)=\frac{b-c}{b+c} \operatorname{Cot} \frac{1}{2} A$

where $C$ is the arbitrary constant; on changing to Cartesian coordinates, we find that the curves are the intersections of the sphere $x^{2}+y^{2}+z^{2}=r^{2}$, and the quadrics

$$
C x^{2}+(C-1) y^{2}+2 \tan \phi_{0} z x-r^{2}=0,
$$

and are therefore sphero-conics.

Finally, it may be remarked that if the earth's magnetic field were free from local irregularities, then the lines of equal magnetic variation (isogonals) would be curves of constant bearing with the points $Z, Z^{\prime}$ at the magnetic poles. The Mercator projection of a curve of constant bearing has four asymptotes, two at each pole, but little more than this can be deduced from the usual magnetic charts.

The Royal Technical College, Glasgow.

\section{A geometrical proof of $\tan \frac{1}{2}(B-C)=\frac{b-c}{b+c} \cot \frac{1}{2} A$}

By A. D. Rossell.

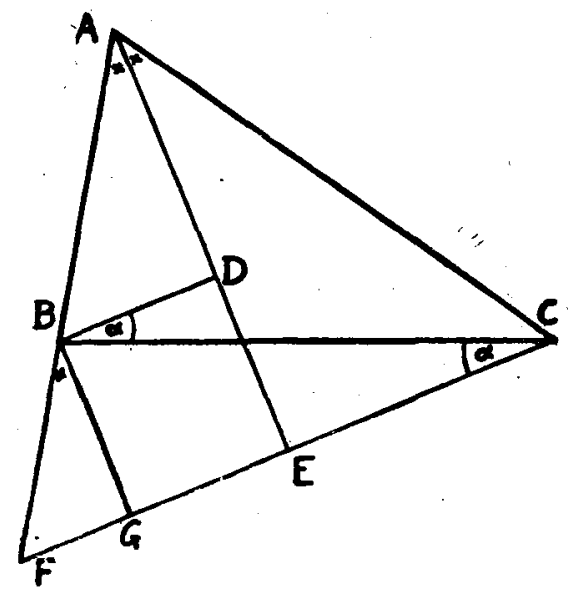

In the figure, $A B C$ is a triangle with $B>C ; A F$ is made equal to $A C, A E$ bisects the angle $A$, and $B D E G$ is a rectangle. It is easily seen that the angles marked $a$ are equal, that $B-a=C+a$, and hence that $\alpha=\frac{1}{2}(B-C)$. Then:

$\tan \frac{1}{2}(B-C)=\frac{B G}{C G}=\frac{B F \cos \frac{1}{2} A}{C E+D B}=\frac{(b-c) \cos \frac{1}{2} A}{b \sin \frac{1}{2} A+c \sin \frac{1}{2} A}=\frac{b-c}{b+c} \cot \frac{1}{2} A$.

\section{Hedgh Street,} Falkirk. 